\title{
COMPLETE METRICS CONFORMAL TO THE HYPERBOLIC DISC
}

\author{
J. BLAND AND MORIS KALKA ${ }^{1}$
}

\begin{abstract}
In this paper we study complete metrics conformal to the hyperbolic disc. We show that any smooth function $K$ bounded between two negative constants is the curvature of such a metric. We also show that if $K \geq 0$ near the boundary, $K$ cannot be the curvature of such a metric.
\end{abstract}

Introduction. Here we consider complete metrics which are conformally equivalent to the hyperbolic metric on the unit disc in $\mathbf{R}^{\mathbf{2}}$. If we denote the hyperbolic metric by $h=d x d y /\left(1-r^{2}\right)^{2}$ and if $g$ is conformally equivalent to $h$, then $g$ is of the form

$$
g=\frac{e^{2 u} d x d y}{\left(1-r^{2}\right)^{2}}
$$

for some function $u$. If we denote the Gauss curvature of $g$ by $K_{g}$, then $u$ satisfies the differential equation

$$
\Delta_{h} u=-1-K_{g} e^{2 u},
$$

where $\Delta_{h}$ is the hyperbolic Laplacian

$$
\Delta_{h}=\left(1-r^{2}\right)^{2}\left(\frac{\partial^{2}}{\partial x^{2}}+\frac{\partial^{2}}{\partial y^{2}}\right)
$$

which henceforth we denote by $\Delta$.

We pose the question: For which smooth functions $K$ can equation $(*)$ be solved so that the resulting metric is complete? We also discuss the uniqueness of the solution for a given $K$.

We can summarize our results as follows:

Existence. Suppose there exist complete metrics $g_{1}, g_{2}$ conformal to $h$ with $g_{1} \leq g_{2}$ and $K_{g_{1}} \leq K_{g_{2}}$. Let $K_{g_{1}} \leq K \leq K_{g_{2}}$ be given. Then there exists a complete metric $g$ with $g_{1} \leq g \leq g_{2}$ and with Gauss curvature equal to $K$. In particular, if $c_{2} \leq K \leq c_{1}<0$, then there is a complete metric uniformly and conformally equivalent to $h$ with curvature equal to $K$. In this case, the metric will also be unique.

We will exhibit examples of complete metrics with curvature tending to $-\infty$ at the boundary, complete metrics with curvature tending to 0 at the boundary, and metrics which have some positive curvature.

Nonexistence. There are no complete metrics conformally equivalent to $h$ with curvature nonnegative in a neighborhood of the boundary.

Uniqueness. If $K$ is bounded between two negative constants, then the solution to $(*)$ is unique. However, there exist functions $K$ which tend to a negative constant

Received by the editors January 22, 1985 and, in revised form, May 24, 1985.

1980 Mathematics Subject Classification. Primary 53C99; Secondary 35J70.

${ }^{1}$ Both authors acknowledge support from the Committee on Research of Tulane University. 
near the boundary and for which there are distinct uniformly equivalent conformal metrics with curvature equal to $K$.

Boundedness. If $K \leq c<0$ in a neighborhood of the boundary, then the solution is bounded from above, while if $K \geq-N$ in a neighborhood of the boundary the solution is bounded below. These follow readily from the local Schwarz lemma [2]. We will also show that if $K \rightarrow 0$ near the boundary, there is no complete metric uniformly and conformally equivalent to $h$ with curvature equal to $K$. The same result holds if $K \rightarrow-\infty$ near the boundary.

Similar results on the existence and uniqueness of solutions have been independently obtained by P. Aviles and R. McOwen [1]; they have also extended their results to the case $n \geq 3$.

\section{Existence.}

THEOREM 1. Suppose $\psi, \phi$ and $K$ are smooth functions on $B=\left\{x \in \mathbf{R}^{2}|| x \mid<\right.$ 1) with the properties that

(i) $\phi \leq \psi$ in $B$, and

(ii) $\Delta \phi \geq-1-K(x) e^{2 \phi}, \Delta \psi \leq-1-K(x) e^{2 \psi}$.

Then there exists $u \in C^{\infty}(B)$ with $\phi \leq u \leq \psi$ such that $\Delta u=-1-K(x) e^{2 u}$.

Proof. Fix a smooth function $\phi \leq g \leq \psi$ on $B$. For $R<1$, set $B_{R}=\{x \in$ $\left.\mathbf{R}^{2}|| \mathbf{x} \mid<R\right\}$. On $B_{R}$ the operator $\Delta$ is uniformly elliptic. Thus by standard elliptic theory (see, for example, $[\mathbf{3}])$ we can find $u_{R} \in C^{\infty}\left(B_{R}\right)$ satisfying

$$
\begin{gathered}
\Delta u_{R}=-1-K(x) e^{2 u_{R}} \quad \text { in } B_{R} \text { and } \phi \leq u_{R} \leq \psi, \\
\left.u_{R}\right|_{\partial B_{R}}=\left.g\right|_{\partial B_{R}} .
\end{gathered}
$$

We show that a subsequence of the $u_{R}$ converges uniformly on compact sets to a smooth solution $u$.

It is clear that $\phi \leq u_{R} \leq \psi$ on $B_{R^{\prime}}$ for all $R>R^{\prime}$. We now proceed to get uniform gradient estimates on $B_{R^{\prime}}$ for all $u_{R}, R \geq R^{\prime \prime}=R^{\prime}+\frac{1}{2}\left(1-R^{\prime}\right)$.

It is well known that the inequality

$$
\left.\left|d_{x, \partial B_{R^{\prime \prime}}}\right| \nabla u_{R}\right|_{0, B_{R^{\prime \prime}}} \leq C\left(\left|u_{R}\right|_{0, B_{R^{\prime \prime}}}+\left|d_{x, \partial B_{R^{\prime \prime}}}^{2} \Delta_{E} u_{R}\right|_{0, B_{R^{\prime \prime}}}\right)
$$

holds, where $C$ is a constant depending only on dimension, $\Delta_{E}$ is the Laplacian with respect to the Euclidean (flat) metric, $|\nabla u|$ is the norm with respect to the Euclidean metric and $d_{x, \partial B_{R}}$ denotes the distance from $x$ to the boundary of $B_{R}$. Applying this to $u_{R}$ for $x \in B_{R^{\prime}}$, one obtains

$$
\begin{aligned}
\frac{1}{2}\left(1-R^{\prime}\right)\left|\nabla u_{R}\right|_{0, B_{R^{\prime}}} & \leq\left|d_{x, \partial B_{R^{\prime \prime}}}\right| \nabla u_{R}||_{0, B_{R^{\prime \prime}}} \\
& \leq C\left(\left|u_{R}\right|_{0, B_{R^{\prime \prime}}}+\left|d_{x, \partial B_{R^{\prime \prime}}}^{2} \Delta_{E} u_{R}\right|_{0, B_{R^{\prime \prime}}}\right) \\
& \leq C\left(\left|u_{R}\right|_{0, B_{R^{\prime \prime}}}+\left|d_{x, \partial B}^{2} \Delta_{E} u_{R}\right|_{0, B_{R^{\prime \prime}}}\right)
\end{aligned}
$$

We note that $d_{x, \partial B}^{2} \Delta_{E} u_{R}$ is uniformly equivalent to $\Delta u_{R}$. Since $u_{R}$ satisfies $(*)$ in $B_{R}$, the right-hand side of the inequality is bounded by

$$
C\left(\left|u_{R}\right|_{0, B_{R^{\prime \prime}}}+\left|-1-K(x) e^{2 u_{R}}\right|_{0, B_{R^{\prime \prime}}}\right)
$$


which in turn is bounded independently of $R$. Thus we can let $R \rightarrow 1$ and we get a uniform $C^{1}$-bound on $u_{R}$ in $u_{R^{\prime}}$ for $R>R^{\prime}+\frac{1}{2}\left(1-R^{\prime}\right)$. Standard elliptic theory now gives us uniform estimates on the $C^{2+\alpha}$ norm of $u_{R}$ independent of $R$.

Let $R_{j}$ be an increasing sequence of numbers $0<R_{j}<R_{j+1}<1$ tending to 1. On $B_{R_{1}}$ the sequence $\left\{u_{R_{j}}\right\}_{j=2}^{\infty}$ is uniformly bounded in ||$_{2+\alpha, B_{R_{1}}}$ and hence has a subsequence $\left(u_{1, j}\right)$ which converges to a solution. On $B_{R_{2}}$, the sequence $u_{1, j}$ are uniformly bounded in the $\left.\right|_{2+\alpha, B_{R_{2}}}$ norm (for $j$ sufficiently large). Hence, we extract a subsequence $u_{2, j}$ which converges uniformly on $B_{R_{2}}$. Continue inductively in this fashion. Then a subsequence of the diagonal sequence will converge uniformly on compact subsets to a solution.

COROLlaRY. Suppose that $c_{1} \leq K \leq c_{2}<0$. Then there exists a smooth function $u$ which solves $\Delta u=-1-K(x) e^{2} u$.

We now give examples of metrics $e^{2 u} h$ which exhibit, in turn, the following properties:

(i) $K>0$ at some points,

(ii) $K \rightarrow-\infty$ as $r \rightarrow 1$,

(iii) $K \rightarrow 0$ as $r \rightarrow 1$.

We will see in $\S 2$ that any metric which exhibits property (ii) or (iii) cannot be uniformly equivalent to $h$. Thus the solutions cannot be bounded.

(i), (iii). Let $\phi=\left(1-r^{2}\right)^{p}$. Then one computes that $\Delta \phi=\phi\left(4 p^{2} r^{2}-4 p\right)$. If $g=e^{2 \phi} h$, then

$$
K_{g}=-(\Delta \phi+1) e^{-2 \phi}=-\left(1+\phi\left(4 p^{2} r^{2}-4 p\right)\right) e^{-2\left(1-r^{2}\right)^{p}} .
$$

If $p=1$, then $g$ is a complete metric with positive curvature at 0 . If $p=-1$, then $g=e^{2 / 1-r^{2}} h$ is a complete metric and $K_{g}=-\left(1+\phi\left(4 r^{2}+4\right)\right) e^{-2 / 1-r^{2}}$ tends to 0 at $r=1$.

(ii) Let $\phi \in C^{\infty}(B)$ have the property that $\phi=C \neq 0$ near $r=0$ and $\phi=$ $-\ln \left(-\ln \left(1-r^{2}\right)\right)$ near $r=1$. Then near $r=1$,

$$
\Delta \phi=4\left[r^{2}\left(\ln \left(1-r^{2}\right)\right)^{-2}+\left(\ln \left(1-r^{2}\right)\right)^{-1}\right]
$$

and if $g=e^{2 \phi} h$,

$$
K_{g}=-\left[1+4\left\{r^{2}\left(\ln \left(1-r^{2}\right)\right)^{-2}+\left(\ln \left(1-r^{2}\right)\right)^{-1}\right\}\right]\left(\ln \left(1-r^{2}\right)\right)^{2}
$$

which tends to $-\infty$.

We see that $g$ is complete since

$$
g_{i j}=(1 / u \ln u)^{2} \delta_{i j}
$$

for $u=\left(1-r^{2}\right)$.

\section{Nonexistence.}

THEOREM 2. Suppose $K \in C^{\infty}(B)$ is nonnegative in a neighborhood of $\partial B$. Then there does not exist any complete metric $g$, conformal to $h$, having curvature $K$.

ProOF. Suppose $g=e^{2 u} h$ is such a metric. Consider the identity mapping $(B, g) \rightarrow(B, h)$. By the local Schwarz lemma [2], the map is metric decreasing, up to a factor. Thus $e^{2 u} /\left(1-r^{2}\right)^{2} \geq C /\left(1-r^{2}\right)^{2}$ and $u$ is bounded below. 
Thus we may assume that $u \geq C$ satisfies $\Delta u=-1-K(x) e^{2 u}$. We denote by $\bar{u}(r)$ the average of $u$ on circles of radius $r$. Since $\Delta$ is rotationally symmetric, $\overline{\Delta u}=\Delta \bar{u}$. As $K$ is nonnegative near the boundary, it follows that $\Delta \bar{u} \leq-1$ near $\partial B$. Thus

$$
\frac{\left(1-r^{2}\right)^{2}}{r} \frac{\partial}{\partial r}\left(r \frac{\partial \bar{u}}{\partial r}\right) \leq-1 .
$$

We may integrate this inequality explicitly to see that $\bar{u} \rightarrow-\infty$. Hence there is a sequence $p_{i} \rightarrow \partial B$ such that $u\left(p_{i}\right) \rightarrow-\infty$, contradicting the lower boundedness of $u$.

Proposition 3. If $K \rightarrow 0$ or $K \rightarrow-\infty$ near $\partial B$, there do not exist any conformal metrics uniformly equivalent to $h$, with curvature $K$. (That is, there do not exist any bounded solutions to $(*)$.)

ProOF. First suppose $K \rightarrow 0$ near $\partial B$ and $g=e^{2 u} h$ has curvature $K$. Thus $\Delta u \rightarrow-1$ near $\partial B$. In particular $\Delta u \leq c<0$ near $\partial B$. By radial averaging as in the proof of Theorem 2 , we see that $u$ is not bounded below.

Now suppose that $K \rightarrow-\infty$ near $\partial B$ and $g=e^{2 u} h$ has curvature $K$. Since $K=-(\Delta u+1) e^{-2 u}$ we see that $\Delta u \rightarrow+\infty$. Using the radial averaging technique again, we see that there is a sequence $p_{i}$ with $u\left(p_{i}\right) \rightarrow \infty$.

REMARK. It is an immediate consequence of the local Schwarz lemma that (i) if $K \leq-\varepsilon$ near $\partial B$, then $u$ is bounded above, and (ii) if $K \geq-N$ near $\partial B$, then $u$ is bounded below.

\section{Uniqueness.}

PROPOSITION 4. Let $c_{1} \leq K \leq c_{2}<0$. Then there exists a unique complete metric $g$ conformal to $h$, with curvature $K$.

PROOF. Existence has already been shown. Thus let $g_{1}=e^{2 u} h$ and $g_{2}=e^{2 v} h$ both have curvature $K$. Then $\Delta(u-v)=-K\left(e^{2 u}-e^{2 v}\right)$. By the remark above, $u$ and $v$ are both bounded. Thus $(u-v)$ is bounded. By the generalized maximum principle [4], there is a sequence of points $p_{k}$ for which $\lim _{k \rightarrow \infty}(u-v)\left(p_{k}\right)=$ $\inf _{B}(u-v)$ and $\lim _{k \rightarrow \infty} \Delta(u-v) \geq 0$. Thus $\lim _{k \rightarrow \infty}\left(-K\left(e^{2 u}-e^{2 v}\right)\right) \geq 0$ implying $\lim _{k \rightarrow \infty}(u-v) \geq 0$. A similar argument shows that $\sup (u-v) \leq 0$. Thus $u \equiv v$.

THEOREM 5. There exists $K$ with the following properties:

(i) $K$ tends to a negative constant near $\partial B$.

(ii) There are two distinct complete metrics $g_{1}=e^{2 u} h$ and $g_{2}=e^{2 v} h$ uniformly and conformally equivalent to $h$ and both having curvature $K$.

Proof. Let $\phi \in C^{\infty}(B)$ satisfy

(i) $\phi \neq 0$ in $B$,

(ii) $\phi \equiv 1$ in a neighborhood of the origin,

(iii) $\phi=\left(1-r^{2}\right)^{1 / 2+\sqrt{3} / 2}=\left(1-r^{2}\right)^{\alpha}$ near $r=1$.

Define $f=\Delta \phi /\left(e^{2 \phi}-1\right)$. Then $f$ is a smooth function which is identically 0 near the origin. We have seen in $\S 1$ that

$$
\Delta\left(1-r^{2}\right)^{\alpha}=\left(1-r^{2}\right)^{\alpha}\left(4 \alpha^{2} r^{2}-4 \alpha\right) .
$$

Thus, near $r=1, f$ is approximately equal to $\left(1-r^{2}\right)^{\alpha}\left(4 \alpha^{2} r^{2}-4 \alpha\right) / 2\left(1-r^{2}\right)^{\alpha}$. By the choice of $\alpha$, we see that $f=1+O\left(1-r^{2}\right)$. 
It is thus possible to find a bounded solution, $v \in C^{\infty}(B)$, of the equation $\Delta v=f-1$. We define $K=-(\Delta v+1) e^{-2 v}$ and $u=v+\phi$.

Then $\Delta v=-1-K e^{2 v}$ by definition and

$$
\Delta u=(f-1)+f\left(e^{2 \phi}-1\right)=(\Delta v+1) e^{2 u} e^{-2 v}-1=-1-K e^{2 u} .
$$

It is easy to check that $K$ tends to a negative constant near $\partial B$.

NOTE ADDED IN PROOF. Because of the normalization involved in our choice of hyperbolic metric $h$, equation $(*)$ should read: $\Delta_{h} u=-4-K_{g} e^{2 u}$. This will not affect any of the results, but it will lead to corresponding changes throughout the paper.

\section{REFERENCES}

1. P. Aviles and R. McOwen, Conformal deformations of complete manifolds with negative curvature, J. Differential Geom. 21 (1985), 269-281.

2. J. Bland, Local boundary regularity of the canonical Kähler-Einstein metric on pseudoconvex domains, Math. Ann. 263 (1983), 289-301.

3. R. Courant and D. Hilbert, Methods of mathematical physics. II, Interscience, New York, 1962.

4. S. T. Yau, Harmonic functions on complete Riemannian manifolds, Comm. Pure Appl. Math. 28 (1975), 201-228.

Department of Mathematics, Tulane University, New Orleans, Louisiana 70118 